\title{
eLS
}

WILEY-

BLACKWELL

\section{Thalidomide and birth defects}

\begin{tabular}{|c|c|}
\hline Journal: & eLS \\
\hline Manuscript ID & A26052.R1 \\
\hline Wiley - Manuscript type: & Standard Article \\
\hline Date Submitted by the Author: & $\mathrm{n} / \mathrm{a}$ \\
\hline \multirow[t]{2}{*}{ Complete List of Authors: } & $\begin{array}{l}\text { Fraga, Lucas; University of Aberdeen, } \\
\text { Diamond, Alex; University of Aberdeen, } \\
\text { Vargesson, Neil; University of Aberdeen, }\end{array}$ \\
\hline & $\begin{array}{l}\text { Thalidomide is a teratogenic drug that caused horrific birth defects when } \\
\text { prescribed as an antiemetic to pregnant women in the } 1960 \text { 's. The most } \\
\text { stereotypical defect is symmetrical limb malformations such as phocomelia, } \\
\text { though ear, eye and internal organ defects are also observed. Thalidomide } \\
\text { was consequently withdrawn from the market. However, Thalidomide has } \\
\text { since been shown to have many beneficial anti-inflammatory and } \\
\text { immunomodulatory effects and is therefore used in a regulated manner in } \\
\text { the treatment against cancers and inflammatory disorders. Sadly, new } \\
\text { cases of babies affected by thalidomide are being born in Brazil, likely due } \\
\text { to medicine sharing. The mechanisms of how thalidomide causes a wide } \\
\text { range of embryonic malformations are becoming clearer; thalidomide is } \\
\text { thought to act through molecules such as cereblon and tubulin and also } \\
\text { affect blood vessel development and cell death, resulting in teratogenesis. } \\
\text { Fully understanding the molecular events induced by thalidomide is } \\
\text { essential if we are to develop a safe but clinically relevant form of the drug. }\end{array}$ \\
\hline
\end{tabular}

\section{SCHOLARONE ${ }^{m}$}


1

10

2 Thalidomide and Birth Defects

$3 \quad$ A26052

4

5

6 Lucas Rosa Fraga*, Alex J Diamond*, Neil Vargesson $\$$

7 School of Medicine, Medical Sciences and Nutrition, Institute of Medical Sciences,

8 University of Aberdeen, Foresterhill, Aberdeen, AB25 2ZD, UK

9

10

11

12

13 Advanced Article

14 * - These authors contributed equally to this Review Article.

15 \$ - Author for correspondence: email: n.vargesson@abdn.ac.uk;

16 nvargesson@gmail.com. Tel: (0)1224 437374

17

18

19 
1 Abstract

2 Thalidomide is a teratogenic drug that caused horrific birth defects when prescribed 3 as an antiemetic to pregnant women in the 1960's. The most stereotypical defect is 4 symmetrical limb malformations such as phocomelia, though ear, eye and internal 5 organ defects are also observed. Thalidomide was consequently withdrawn from the 6 market. However, Thalidomide has since been shown to have many beneficial anti7 inflammatory and immunomodulatory effects and is therefore used in a regulated 8 manner in the treatment against cancers and inflammatory disorders. Sadly, new 9 cases of babies affected by thalidomide are being born in Brazil, likely due to 10 medicine sharing. The mechanisms of how thalidomide causes a wide range of 11 embryonic malformations are becoming clearer; thalidomide is thought to act through 12 molecules such as cereblon and tubulin and also affect blood vessel development 13 and cell death, resulting in teratogenesis. Fully understanding the molecular events 14 induced by thalidomide is essential if we are to develop a safe but clinically relevant form of the drug.

\section{Key Words}

angiogenesis, cell death, Cereblon, reactive oxygen species, time sensitive window, mechanisms of teratogenesis, chicken embryo, zebrafish embryo

\section{Key Concepts}

- Thalidomide was used between 1957 and 1961 as a 'safe' treatment for morning sickness, but was withdrawn after it was found to cause severe birth defects.

- Thalidomide has since been shown to possess anti-inflammatory, antiangiogenic and anti-proliferative properties. 
- Thalidomide is now used, under strict regulations, to treat human inflammatory disorders and cancer.

- Thalidomide causes embryonic damage in a short time-sensitive window between day 20 and 36 post-fertilisation in humans.

- Thalidomide causes damage to the majority of the body tissues, amongst the most common and stereotypical damage is to the limbs.

- Effects of thalidomide can vary dependent on the species exposed, some species being more sensitive to the drug than others.

- Evidence supports blood vessels as a primary target of thalidomide.

- Possible other pathways involved in thalidomide-induced embryopathy are oxidative stress induction, cell death and binding to Cereblon.

- Cereblon acts as a target of thalidomide for treatment of multiple myeloma in adult humans. 
11 History of Thalidomide

2

3 Thalidomide [a-(N-phthalimido) glutaramide] was synthesised by Chemie 4 Grunenthal, in Germany, and introduced onto the market in 1957 as a "safe", non5 addictive, over-the-counter sedative (Vargesson, 2015). The drug was marketed 6 across 46 countries and was also sold as an effective antiemetic for pregnant 7 women suffering morning sickness (Franks et al., 2004; Vargesson, 2009; 8 Vargesson, 2013; Vargesson, 2015).

9 Following the release of thalidomide, reports of an increase in the occurrence of severe and rare birth defects began surfacing (McCredie, 2009; Vargesson, 2013; Vargesson, 2015). The most striking defect was phocomelia of the limbs (where distal structures of the limb remain whereas proximal structures are lost or reduced), though some babies presented with amelia (no limb structures exist). A wide range of damage to the limbs could be observed as well as damage to many other body systems (see Section 3) (Lenz and Knapp, 1962; Ruffing, 1977). Damage to the ears, eyes, genitalia, heart, gastrointestinal tract and kidneys was also reported (Smithells and Newman, 1992; Vargesson, 2009; Vargesson, 2013; Vargesson, 2015). The range and severity of damage to many babies across Europe confused clinicians at the time. It was not till two clinicians, McBride in Australia and Lenz in Germany, independently concluded in 1961 that the children with these birth defects were born to mothers who had consumed thalidomide (McBride, 1961; Lenz, 1962; Lenz, 1988). The drug was withdrawn from the worldwide market on 30 Nov 1961 (Matthews and McCoy, 2003; Vargesson, 2013). The consumption of thalidomide during pregnancy was confirmed as the cause of birth defects since there was an almost complete loss of such defects from 1962 onwards (Lenz, 1988; Smithells and Newman, 1992; Vargesson, 2013; Vargesson, 2015). However, it is estimated that at least 10,000 children were born with deformities resulting from thalidomide exposure (Smithells and Newman, 1992; Vargesson, 2009). Thalidomide was not approved for use in America during the 1957-1962 thalidomide disaster: Dr Frances Kelsey, working for the US Food and Drug Administration (FDA), doubted its safety after reports of peripheral neuropathy in patients (Matthews and McCoy, 2003; Franks et al., 2004; Vargesson, 2013; Vargesson, 2015). If thalidomide had been released in 
1 the US, there may have been a significantly higher number of cases of birth defects, 2 as seen in Europe, Canada, Australia and Japan.

3 Thalidomide underwent a rebirth in 1965 after studies proved its effectiveness as a 4 treatment for erythema nodosum leprosum (ENL), a complication of leprosy 5 (Sheskin, 1965). Following this, thalidomide was licensed in Mexico, Brazil and later 6 in the US for the use in the treatment of ENL (Franks et al., 2004) and in 2006 for 7 treatment of multiple myeloma (MM) (Latif, 2012).

8 Programs now administer the use of thalidomide under strict guidelines where 9 women prescribed the drug are required to use birth control and take regular 10 pregnancy tests. When these guidelines are followed, no occurrence of thalidomide 11 embryopathy has been reported (Uhl et al., 2006). However, tragically, in Brazil, children are still being born with thalidomide embryopathy where the drug is used to effectively treat leprosy. This is likely due to a culture of sharing medicines as a result of people living so far away from hospitals, misinterpretation of the drug, and pregnant women taking it whilst suffering from leprosy (Vianna et al., 2013; Vargesson, 2013). Considering the beneficial properties of thalidomide there is the possibility of increased use and a concern for a further potential increase in the frequency of thalidomide-induced birth defects. Despite research efforts the mechanisms of thalidomide-induced embryopathy are not fully understood. Continuing research is vital in the mission to synthesise a safe, clinically relevant form which is non-teratogenic, i.e. does not cause birth defects.

\section{Biochemistry of thalidomide}

Thalidomide is a derivative of the non-essential amino acid glutamic acid (Franks et al., 2004). The structure consists of a glutarimide ring, pthalimido ring and contains an asymmetric carbon atom (Figure 1). The presence of the chiral carbon allows thalidomide to exist in two, interchangeable states, or enantiomers $(S(-))$ and $R(+))$, within the body. One state is thought to be the causative 'teratogenic' state $(S(-))$, and the other the 'sedative' state $(R(+))$. Since the drug can switch states within the body, it is not conceivable to prescribe just the 'safe', 'sedative' version. Thalidomide can broken down in to its active state by the liver enzyme cytochrome P450 and has 
1 a half-life of 6-12 hours. Thalidomide can also rapidly hydrolyse in bodily fluids

2 (Franks et al., 2004; Vargesson, 2009; Vargesson, 2013; Vargesson, 2015).

\subsection{Pharmacological Properties of Thalidomide}

4 Further research in to the mechanism of thalidomide action has revealed a wide, 5 diverse range of functions. As well as being anti-inflammatory and 6 immunomodulatory, thalidomide is also anti-angiogenic and has anti-proliferative 7 activities (D'Amato et al., 1994; El-Aarag et al., 2014). Through these properties

8 thalidomide has been identified as an effective treatment for a number of adult 9 conditions. Indeed since the discovery in 1965 that thalidomide can be beneficial as 10 an anti-inflammatory drug to treat ENL, studies have recognised its clinical purpose 11 as treatment for multiple myeloma (MM), cancers, Behcet's disease, gastrointestinal 12 disorders, rheumatological disorders, hereditary hemorrhagic terangiectasia (HHT), 13 lupus, idiopathic pulmonary fibrosis, HIV and diabetic retinopathy (Franks et al., 14 2004; Vargesson, 2013; Vargesson, 2015).

\subsubsection{Antiangiogenic actions}

16 Thalidomide has the ability to inhibit angiogenesis, the formation of new and 17 remodelling blood vessels. This action was first reported by using rabbit and rodent 18 cornea assays to show that thalidomide inhibits fibroblast growth factor (FGF)19 induced angiogenesis (D'Amato et al., 1994). In chicken embryos thalidomide inhibits nitric oxide (NO), an important molecule for endothelial cell function and protection of blood vessels (Siamwala et al., 2012; Majumdar et al., 2009; Tamilarasan et al., 2006; see also DOI: 10.1002/9780470015902.a0003390.pub2). NO is required for normal limb development since it promotes angiogenesis and reduces oxidative stress, therefore inhibition by thalidomide leads to limb malformations. Indeed, thalidomide affected chicken and zebrafish embryos can be rescued by NO (Siamwala et al., 2012). Additionally, thalidomide inhibits NO-induced endothelial cell migration as well as interfering with normal actin polymerisation patterns. This prevents cells forming tubes, thereby inhibiting angiogenesis at the cellular level (Tamilarasan et al., 2006; Vargesson, 2013; Vargesson, 2015).

Thalidomide also induces degradation of Tumor Necrosis Factor- $\alpha$ (TNF $\alpha$ ) mRNA, a pro-angiogenic cytokine, suggesting another mechanism by which thalidomide 
1 inhibits angiogenesis (Moreira et al., 1993). Thalidomide has been demonstrated to 2 reduce the vascular hemorraging and malformations in patients suffering from HHT 3 by inhibiting angiogenesis and through recruitment of mural cells, known to decrease 4 endothelial cell migration and proliferation, causing early maturation of blood vessels 5 (Lebrin et al., 2010; Figure 2). In zebrafish embryos, thalidomide reduces VEGF 6 receptor function (Yabu et al., 2005; Vargesson, 2013; Vargesson, 2015). In chicken 7 embryos, exposure of early blood vessels to thalidomide results in a breakdown of 8 vascular formation (Tamilarasan et al., 2006). Antiangiogenic analogs of thalidomide, 9 as opposed to anti-inflammatory analogs, cause limb defects (Therapontos et al., 10 2009). The antiangiogenic actions of the drug make it a promising therapeutic agent 11 for the treatment of tumours, since it can prevent their early vascularisation 12 (Therapontos et al., 2009).

\subsubsection{Anti-proliferative actions}

The anti-proliferative effects of thalidomide are independent of its immunomodulatory activities in hematologic malignancies. Thalidomide reduces proliferation of cancerous MM cells that are resistant to standard chemotherapy (Melcherd and List, 2007). Myeloma cells are targeted by thalidomide through several mechanisms including activation of antitumor immunity and exertion of antiangiogenic effects. The treatment of MM patients with thalidomide improves their survival rate, but the exact way in which thalidomide achieves this is not fully understood. Current studies are pointing to a molecular pathway targeted by thalidomide to combat MM which involves Cereblon, Ikaros and Aiolos proteins. Cereblon is part of an E3 ubiquitin ligase complex with the proteins Damaged DNA binding protein 1 (DDB1), Cullin-4A (CUL4A), and regulator of Cullin1 (Roc1). This complex tags proteins with ubiquitin, labelling them for proteolysis, and is therefore important for the regulation of protein expression (Stewart, 2014; Ito et al., 2010; Ito et al., 2011). After binding to thalidomide, Cereblon protein is inactivated, resulting in the rapid ubiquitination and degradation of Ikaros and Aiolos. Both proteins are transcription factors that in normal conditions regulate $\mathrm{T}$ and $\mathrm{B}$ cell development. High degradation of Ikaros and Aiolos increase the Interleukin-2 (IL) levels and decreases TNFa levels (Stewart, 2014) (Figure 2). In addition, a correlation exists between low amounts of Cereblon in MM cells, clinical drug resistance and poor survival outcomes (Schuster et al., 2014; Stewart, 2014). Thalidomide reduces expression of $T N F \alpha, N F-K B, I L-6$ and -8 
1 and Vascular Endothelial Growth Factor (VEGF) proteins which are related to tumour

2 cell survival, proliferation, inhibition of apoptosis and resistance to therapy (Latif et 3 al., 2012).

$6 \quad$ 2.1.3 Anti-inflammatory actions

7 Thalidomide exhibits immunomodulatory and anti-inflammatory effects through TNF $\alpha$ 8 mRNA degradation, Nuclear Factor-kappa-B (NF-kB) regulation and 9 Cyclooxygenase-2 (COX2) inhibition (Moreira et al., 1993; Vargesson, 2015). 10 Inducing TNFa mRNA degradation supresses the activation of interleukins and 11 cytokines by monocytes and macrophages. ENL patients present with high levels of 12 TNFa, which reduce with thalidomide treatment (Sampaio, 1993; Vargesson, 2013). 13 The effects of thalidomide on TNFa is beneficial when treating other autoimmune 14 diseases which arise through an overproduction of inflammatory cytokines (Latif et 15 al., 2012). A key regulator of the expression of cytokines, including $T N F \alpha$, is 16 transcription factor NF-kB. Thalidomide selectively blocks TNFa and hydrogen 17 peroxide-induced NF-kB activation, interfering with $T N F \alpha$ expression and other 18 inflammatory molecules such as IL-8 (Majumdar et al., 2002). Cytokine COX-2, 19 involved in both inflammatory response and cancer growth, is also suppressed by 20 thalidomide (Melcherd and List, 2007).

21 In addition to these actions which are the basis for some of thalidomide's clinical 22 applications the drug can also induce cell death (Knobloch et al., 2007) as well as 23 reactive oxygen species (ROS) (Parman et al., 1999). The multiple and varied 24 actions of the drug, in part, explain why it has been so difficult to determine the 25 precise mechanism underlying thalidomide induced teratogenesis. As we will see 26 current viewpoints favour the antiangiogenic action of the drug as a major cause of 27 teratogenesis.

\subsection{Thalidomide acts in a time sensitive window}


1 Thalidomide induces damage to the embryo in a time-sensitive window between 2 days 20 and 36 post-fertilization (Figure 3) (Vargesson, 2009; Vargesson, 2015).

3 The timing of damage was determined through interviews with mothers who had 4 taken thalidomide, providing data to identify a correlation between when thalidomide 5 was taken and the resulting malformations (Lenz and Knapp, 1962; Ruffing, 1977; 6 Smithells and Newman, 1992). Since the symptoms of typical morning sickness 7 coincide with a period of rapid development and embryogenesis, thalidomide was 8 taken at a time when countless cell divisions, growth, migration, differentiation and 9 organogenesis are occurring. Exposure to thalidomide interfered with major 10 developmental events, triggering the defects seen in thalidomide embryopathy 11 (Vargesson, 2013). Miscarriage results if the drug is taken before the time-sensitive 12 window (Vargesson, 2015), however it is not known whether exposure to thalidomide after day 36 results in obvious embryonic defects. The babies identified for study and maternal interview had mainly outward, visible defects and so if damage was only obvious later in life, it was not noted. Therefore exposure to thalidomide after the for any embryo to be unharmed following consumption of just one tablet (Smithells and Newman, 1992). Indeed, it is estimated that one $50 \mathrm{mg}$ tablet is sufficient to cause birth defects in at least $20-50 \%$ of embryos exposed to thalidomide during the time-sensitive window (McBride, 1961; Lenz, 1962; Smithells and Newman, 1992; Vargesson, 2009; Vargesson, 2013; Vargesson, 2015)

\subsection{Thalidomide Embryopathy}

Although almost any organ can be affected by thalidomide, the type of malformations observed are dependent on the day of thalidomide intake (Table 1; Figure 3) (Lenz and Knapp, 1962; Ruffing, 1977; Smithells and Newman 1992; Vargesson, 2015). The multi-tissue damage seen is referred to as thalidomide embryopathy (Table 1) where bilateral, symmetrical limb malformation is the most stereotypical defect, but many other body systems are damaged too (Newman, 1986; Smithells and Newman, 1992). Furthermore, thalidomide embryopathy has also been termed thalidomide syndrome, as the damage seen is a collection of damage often occurring independently in other human conditions (Newman, 1986; Smithells and Newman, 1992; Vargesson, 2009; Vargesson, 2013) (see also DOI: 10.1002/9780470015902.a0025686). 
$1 \quad 3.2 .1$ Limb Damage

2 Phocomelia is the most striking limb malformation associated with thalidomide

3 embryopathy, the most severe form of which being the absence of any long bones.

4 The majority of thalidomide survivors have limb defects, ranging from amelia (no

$5 \mathrm{limb}$ ) to triphalangeal thumb and including radial dysplasia, and phocomelia. The

6 majority of limb anomalies seen in thalidomide survivors are reduction events and

7 typically bilateral in nature (Table 1). The thumb is the first bone to be affected,

8 followed by the radius, humerus and ulna (Lenz and Knapp, 1962; McCredie, 2009;

9 Smithells and Newman, 1992; Vargesson, 2013). Lower limb defects are less

10 commonly seen. Shoulder and hip joints can, be weaker in thalidomide survivors and

11 the hip and pubic bones may be missing (Vargesson, 2013).

$12 \quad 3.2 .2$ Ear and Eye Damage

13 Ears and eyes develop around the same time as the limbs in the embryo and so are 14 targeted during the thalidomide time-sensitive window (Figure 3). Complete absence 15 of the eyes, small eyes and poor vision are all reported defects. Unlike limb defects, 16 eye defects can occur unilaterally. Ear defects usually occur bilaterally and in 17 conjunction with eye defects and facial palsies. Malformations range from absence of 18 the ear (anotia), resulting in deafness, to elements of the outer ear remaining 19 (microtia) (Vargesson, 2013; Vargesson, 2015).

\subsubsection{Facial and Neural Damage}

21 Facial muscles and nerves can be damaged by thalidomide and lead to facial palsy 22 or asymmetry. A stereo-typical sign of thalidomide exposure is an enlarged facial 23 naevus at birth, usually on the forehead, though this is no longer visible by three 24 years of age (Vargesson, 2013). Irregular teeth, cleft palate and small noses are 25 additional defects seen in thalidomide survivors. A second consequence of nerve 26 damage by thalidomide during development is an increased occurrence of epilepsy 27 and autism later in life (Smithells and Newman, 1992; Miller et al., 2005).

\subsubsection{Internal Organ Damage}

29 The frequency of internal organ defects is difficult to define since they are not 30 obviously apparent and may not present during childhood. Only the most noticeable 
1 defects will have been recorded during the 1960s. The heart, kidney, gastrointestinal and urinary tracts and genitalia can all be affected by exposure to thalidomide (Lenz and Knapp, 1962; Ruffing, 1977; Smithells and Newman, 1992). Heart malformations can occur with pulmonary stenosis and patent duct arteriosus and are thought to be the main cause of miscarriages or postnatal deaths suffered after intake of thalidomide. Kidney defects include rotated, hypoplastic and ectopic kidneys. Internal and external genital defects as well as urinary tract defects are also seen. Testicular absence or malformations in males and abnormalities of the uterus in females are known defects (Lenz and Knapp, 1962; Ruffing, 1977; Smithells and Newman, 1992; Vargesson, 2013; Vargesson, 2015).

The true scale of the number of affected embryos and/or the range of defects caused

\section{How does Thalidomide Cause Damage To The Embryo?}

\subsection{Thalidomide effects are species dependent}

Initial studies by Grunenthal, who invented and marketed the drug, tested thalidomide on rodents, where no defects were detailed or described. Questions remain about the precise testing carried out, but Grunenthal say they carried out testing that was typical of the day. The drug was considered safe and approved for use. After thalidomide was withdrawn from the market it was actually found to act in many species including humans, primates, rabbits, marsupials, zebrafish and chickens (Stephens, 2009; Vargesson, 2013). 
1 Rodents are sensitive to thalidomide but much less so than other organisms, and are 2 affected by much higher doses (DiPaolo et al., 1964; Parkhie and Webb, 1983; 3 Vargesson, 2013). The reason for this species sensitivity difference is unclear. 4 Thalidomide is able to inhibit angiogenesis in mice and rat aortic ring cultures, so 5 although rodents are not insensitive to the drugs mechanisms, there may be aspects 6 such as different rates of metabolism which offer them protection (Lu et al., 2004). 7 Indeed, incubation of thalidomide with rodent liver cytochrome enzymes results in 8 lower angiogenic activity than if incubated with human or rabbit enzymes (Marks et al 9 2002). Clearance of the drug is also much faster in mice compared to humans, so 10 teratogenic forms may not exist for as long (Lu et al., 2004; Vargesson, 2013). 11 Differences in the length of gestation between rodents and humans could also be a factor in predisposition of sensitivity to thalidomide.

Among the mammals, primates are considered the best model to study thalidomide embryopathy giving phenotypes that most similarly reflect those seen in humans (Ema et al., 2010; Vargesson, 2013; Vargesson, 2015). However primates present ethical and practical challenges including low offspring numbers, long gestation times and are costly to work with. Studies in non-human primates have shown characteristic limb reduction malformations, ranging from amelia to phocomelia, and defects in the tail and genitalia (Ema et al., 2010). Rabbit model studies identified a range of defects similar to those found in humans, including limb and internal organ defects (Fratta, 1965). Rabbits are therefore one of the most reliable models used to demonstrate the teratogenic effects of thalidomide. Regarding non-mammalian models, thalidomide is toxic to Xenopus and exposure causes teratogenic effects (Fort et al., 2000). Chicken and zebrafish embryos are excellent for studying thalidomide embryopathy since they develop rapidly and provide easy access to follow development (Stephens, 2009; Vargesson, 2009; Vargesson, 2013). Since these models are perfect for drug screening studies, the effect of thalidomide upon their development is well established making these animal models excellent for the study of thalidomide teratogenicity. In the chicken embryo, thalidomide causes limb and eye defects (Knobloch et al., 2007; Stephens, 2009; Therapontos et al., 2009; Ito et al., 2010; Mahony et al., 2013; Siamwala et al., 2012). In Zebrafish, embryonic 32 fins and eyes are affected (Ito et al., 2010, Mahony et al., 2013; Yabu et al., 2005). In humans thalidomide affects the development of embryos in a time-sensitive manner. 
1 This is also true for other animals, so embryos will be most sensitive to thalidomide 2 during a particular window of development (Stephens, 2009; Therapontos et al., 3 2009; Ito et al., 2010; Mahony et al., 2013). Thalidomide also exhibits intra-species 4 specificity; of eight dizygotic twin pairs examined during the 1960s thalidomide 5 disaster in Brazil, only four pairs were born with the same malformations (Schmidt 6 and Salzano, 1980). Drug distribution, metabolism and the genetic background of 7 each species, strain or individual must be taken in account.

\subsection{Morphological and Molecular Actions of Thalidomide Teratogenicity}

More than 30 theories attempting to explain the mechanisms of thalidomide teratogenesis have been postulated since the 1960s, though most cannot be backed include actions on DNA, bone cells, integrins and many others. Explanations need to address the range of defects seen in thalidomide embryopathy and how the timesensitive window of exposure affects all tissues. Three of the most widely accepted theories are (i) the antiangiogenic action of the drug; (ii) the drugs ability to induce reactive oxygen species (ROS) and cell death; (iii) thalidomide binding to Cereblon.

\subsubsection{Blood Vessels as Targets of Thalidomide}

Blood vessels supply oxygen and nutrients to growing tissues so are essential for embryonic development. It is established that loss or disruption of blood vessels during embryogenesis can lead to death or embryonic malformations (Vargesson, 2003; Vargesson, 2013). It was postulated that limb defects might be caused by the antiangiogenic effect of thalidomide (D'Amato et al., 1994). Indeed damage to vessels can cause limb defects in chicken embryos (Vargesson and Laufer, 2001; Vargesson, 2003; Vargesson, 2009). Studies in chicken embryos have further demonstrated that thalidomide affects angiogenesis even before the expression of some signalling molecules essential for limb development, such as FGFs (Therapontos et al., 2009).

Thalidomide can be broken down in to various by-products, and a large number of structural analogs of thalidomide can be synthesised. This is invaluable to help understand drug function and actions and also determine which characteristic of the 
1 drug is the cause of teratogenesis. The production of CPS49, an antiangiogenic

2 analog, has shed light on the method of teratogenesis. Blood vessels are destroyed

3 within one hour of exposure to CPS49 in an E2.5 chicken embryo, with phocomelia 4 presenting 7 days later (Therapontos et al., 2009; Vargesson, 2009). Cell death is 5 observed after application of CPS49, as well as loss of Fgf8 and Sonic Hedgehog 6 (Shh) expression, both key regulators of limb development and outgrowth.

7 Thalidomide has also been shown to induce cell death and cause the loss of limb 8 signalling events in chicken embryos (Knobloch et al., 2007). Studies indicate that 9 CPS49 destroyed newly forming vessels without a smooth muscle coat. Smooth muscle protects vessels and prevents angiogenesis. In-vitro studies demonstrated that smooth muscle negative vessels undergoing angiogenesis were destroyed but mature, smooth muscle positive vessels were unharmed (Theraponotos et al., 2009). CPS49 also disrupts blood vessels in zebrafish embryos and both CPS49 and thalidomide inhibit the actin cytoskeleton of vascular cells in-vitro (Therapontos et al., 2009; Tamilarasan et al., 2006; Lebrin et al., 2010).

\subsubsection{Reactive Oxygen Species (ROS) and Cell Death}

The production of ROS in embryos causes oxidative stress, cell death and is upregulated in presence of thalidomide (Vargesson, 2013; Vargesson, 2015). Oxidative stress is required for cell-death-dependent thalidomide embryopathy; therefore this model could explain damage to limbs and other tissue. If thalidomide increases production of ROS, this will lead to cell death in affected tissues, causing defects. The function of redox-sensitive NF-KB is also affected by oxidative stress. $\mathrm{NF}-\mathrm{KB}$ is a transcription factor important for limb development, and thalidomide diminishes its ability to bind to DNA promoter targets. This alters expression of Fgf8, Fgf10 and Bone Morphogenetic Proteins (BMP) (Hansen and Harris, 2004), important genes in the process of limb development. Indeed, it has been shown that thalidomide exposure results in upregulation of $B m p-4,-5$ and -7 expression in chicken embryos (Knobloch et al., 2007). However, just how thalidomide induces ROS and/or cell death in a time-sensitive and tissue specific manner is unclear, though it could be a secondary effect to the loss of blood vessels. Considering that oxidative stress is a physiological process and occurs during embryogenesis, how it causes tissue specific damage is unknown. It is understood that NF-KB can negatively regulate BMP signalling which could explain, in part, why limbs are 
1 affected by thalidomide through oxidative stress. How the other tissues are affected

2 and how the range of damage is caused remains unclear.

\section{4.2.3 Cereblon and E3 Ubiquitin-ligase Complex}

4 Thalidomide is proposed to initiate teratogenesis by binding Cereblon, preventing 5 establishment of the E3 ubiquitination complex and consequently causing mis-

6 regulation of developmental signalling molecules (Ito et al., 2010; Ito et al., 2011;

7 Stewart et al., 2014; Vargesson, 2015).

8 In adult humans the Cereblon (CRBN) gene, conserved in species including plants 9 and invertebrates (Higgins et al., 2004), is expressed in several tissues such as the 10 testis, spleen, liver, pancreas, lung and skeletal muscle (Xin et al., 2008). Cereblon 11 was identified as a primary binding target of thalidomide (Ito et al., 2010), supported 12 by results showing mutations preventing the binding between Cereblon and 13 thalidomide suppressed limb loss in chicken embryos (Ito et al., 2010). In addition, 14 through inhibiting the translation of Cereblon mRNA, in zebrafish embryos, some 15 phenotypes were found that appeared similar to those seen in thalidomide treated embryos, though not with the range or severity of damage seen in human thalidomide embryopathy (Ito et al., 2010). Furthermore, Cereblon loss-of-function mice appear normal and unharmed (Lee et al., 2013). Data suggests a participation of Cereblon in thalidomide embryopathy; however how thalidomide binding to Cereblon causes the damage, the range of damage and in a time sensitive manner is unclear, as is the precise role/function of Cereblon in normal embryonic development.

Thalidomide binding to Cereblon has been shown to mediate thalidomide's beneficial anti-inflammatory and anti-myeloma actions in adult and diseased tissues (Figure 2). The downstream targets of Cereblon-Thalidomide binding relating to teratogenesis, however, are not known.

\subsubsection{Tubulin}

Through the use of an antiangiogenic thalidomide analog, 5HPP-33, biochemical and computational assays have shown the affinity of 5HPP-33 to bind tubulin. In addition, 5HPP-33 causes depolymerisation of microtubules and affects rebuilding of mitotic spindles, interfering with the alignment of chromosomes at metaphase (Rashid et al., 
1 2015). Changes in actin and microtubule cytoskeleton cause actin stress fibre and 2 microtubule depolymerisation, altering cell migration and proliferation. Thalidomide 3 exposure to human umbilical vein endothelial cells (HUVECs) results in a disruption 4 of actin cytoskeleton (Tamilarasan et al., 2006), and CPS49 affects migration and 5 cytoskeletal organisation of endothelial cells (Therapontos et al., 2009).

6 These studies provide evidence that tubulin may be a target of thalidomide 7 preventing angiogenesis, leading to cell death of tissues causing thalidomide 8 teratogenesis.

\subsubsection{Soluble Guanyl Cyclase and Nitric Oxide}

Thalidomide has also been shown to potentially interact with soluble guanylyl cyclase (sGC). SGC stimulation by NO leads to production of cyclic guanosine monophosphate (cGMP) which is involved in several cellular processes, including apoptosis, vasodilation and blood flow increase through the control of vascular smooth muscle (Majumder et al., 2009, Siamwala et al., 2012). Experiments in HUVEC cultures showed that thalidomide exposure reduced cGMP levels, causing failure of angiogenesis. This phenotype can be reversed by inducing an increase in sGMP levels (Majumder et al., 2009).

Moreover, thalidomide has been shown to exert effects through alterations in NOmediated endothelial cell migration and apoptosis (Tamilarasan et al., 2006, Siamwala et al., 2012). Assays in chicken embryos show increasing NO may rescue thalidomide teratogenicity (Tamilarasan et al., 2006, Majumder et al., 2009, Siamwala et al., 2012).

\subsubsection{Genetic Studies}

Many other gene expression patterns have been shown to be altered following thalidomide exposure in chicken, zebrafish and non-human primate studies including, for example, Shh, Fgf8 and Integrins (Vargesson, 2009; Vargesson, 2015). How these fit into the molecular pathway/s altered by thalidomide is unclear. Furthermore, studies looking at differential gene expression after direct thalidomide exposure have been carried out using microarray techniques in monkey embryos 
1 and in human and mouse embryonic stem cells. Expression levels of around 2000 2 genes were found to be altered following thalidomide exposure including those 3 involved in cell differentiation, development, metabolism, cytoskeleton organization, 4 limb and heart development and the immune response (Gao et al., 2014; Gao et al., 5 2015; Ema et al., 2010, Meganathan et al., 2012). Some of these changes may be 6 primary, secondary or even tertiary. Indeed, the precise molecular pathway/s 7 influenced by thalidomide remain to be fully determined. The possibility that there 8 may be more than one direct molecular target and pathway affected by thalidomide 9 is plausible.

A genomic study, carried out in human thalidomide affected patients, aimed to assess if a potential genetic susceptibility to thalidomide embryopathy exists by analysing the endothelial Nitric Oxide Synthase gene in thalidomide survivors and non-thalidomide affected individuals. It was observed that alleles relating to a reduced production of $\mathrm{NO}$ are found more frequently in thalidomide subjects. This not only reinforces the involvement of $\mathrm{NO}$ in thalidomide embryopathy but also the role for angiogenesis in thalidomide teratogenesis (Vianna et al., 2013).

\section{Conclusion}

Despite numerous studies and recent advances in our understanding, the mechanisms that result in thalidomide embryopathy are still not completely known. Actions upon blood vessels, induction of cell death and involvement of several gene targets including Cereblon and tubulin are all involved. Just how thalidomide exposure causes changes in molecular pathways and any interrelation among these pathways is unclear. Indeed multiple pathways may be affected to cause the different tissue specific damage. Currently blood vessels as a primary target tissue of thalidomide, which locally induces ROS and cell death in affected tissues, is a strongly favoured teratogenic mechanism of action of thalidomide (Vargesson, 2013; Vargesson, 2015; Figure 4).

Thalidomide was used to treat a range of conditions, including morning sickness, which typically occurs between week 4 and week 12 (although timing and severity can vary between women). Between weeks 4 and 9 major events in embryology 
1 occur along with major cell signalling events, massive cell migration and tissue

2 morphogenetic events. As we have outlined, angiogenesis and vascularisation is an 3 essential step in tissue formation, outgrowth and maintenance. Smooth muscle 4 negative vessels undergo rapid angiogenic changes and migration. Disruption of 5 vessels or loss of vessels in forming tissues could result in cell death and localised 6 ROS activity tissue loss with interrupted signalling devastating rapidly growing 7 tissues and causing malformations. For example, phocomelia in the limbs could 8 occur as vessels are prevented from vascularising the limb, which then starts to 9 undergo cell death, loss of gene expression or gene misexpression. As the activity of 10 the drug wears off, the remaining cells can be vascularised and undergo proliferation 11 and the developing limb gene signalling pathways recover but as too few cells 12 remain, only distal structures develop (Therapontos et al., 2009; Vargesson, 2009; 13 Vargesson, 2015). Appearance of secondary cell types and their development into tissues, for example nerves, muscles and bones, will then be altered as the limb tissue is malformed or even missing (Vargesson, 2013; Vargesson, 2015).

By around week 9 the major tissues are formed and vasculature is also maturing through recruitment of smooth muscle, with reduced angiogenesis. Exposure to thalidomide does not appear to result in outwardly visible malformations after this stage. However, the fact that thalidomide acts in an antiangiogenic manner both in the early embryo and the adult suggests late embryonic exposure could damage physiological function of the internal organs as they mature and enlarge, since tissue expansion requires angiogenesis. The framework of thalidomide embryopathy as described above (Figure 4; and in further detail in Vargesson, 2015) is a good explanation for thalidomide-induced damage to the tissues. It can explain the range of damage and time sensitive nature of the induced damage. Malformations occur dependent upon the maturity of blood vessels and whether they are undergoing angiogenesis, and the chance of defects presenting is reduced as tissues and vessels mature (Therapontos et al., 2009; Vargesson and Laufer, 2001; Vargesson, 2013; Vargesson, 2015).

Challenges do remain, and these include to understand which molecular pathway, or multiple pathways, are affected by thalidomide to cause teratogenesis. We know several molecular targets of thalidomide, Cereblon and tubulin, and know many other gene profiles can be changed following thalidomide exposure. However, just how 
1 thalidomide binding to these targets results in embryopathy is unclear.

2 Understanding the molecular pathways and elucidating any other candidate targets 3 may shed light on novel roles for genes and help to understand how birth defects 4 can be prevented. In addition, determining if a form or analog of thalidomide can be 5 produced with the clinical benefits (for example, an analog that will still treat leprosy) 6 but without the side effect of birth defects, is a significant and essential challenge 7 especially given the new generation of thalidomide affected children in Brazil (Beedie 8 et al., In Press; Vargesson, 2015). Structural variants of thalidomide, for example, 9 Lenalidomide and Pomalidomide, function slightly differently and are used clinically 10 to treat inflammatory diseases and cancer, though with some species-specific 11 teratogenic side-effects (Vargesson, 2013; Vargesson, 2015). Can a form of the drug 12 be made or found that retains clinical relevance but without the drugs side-effects?

13 Great strides in our understanding of thalidomide-induced embryopathy have been 14 made in the recent few years. Thalidomide's use for treating inflammatory disorders 15 in adult humans has increased interest in the drug and other uses for it. In addition, 16 folllowing the birth of recent thalidomide survivors in Brazil interest in determining the teratogenic mechanisms of the drug has also increased. Hopefully it will only be a matter of time before all the mechanisms this drug uses are finally determined and a safe form can be developed.

\section{Acknowledgements}

Apologies to the many papers we were unable to cite, due to space constraints. We thank Lynda Erskine, Shaunna Beedie and Chris Mahony for helpful discussions. Lucas Rosa Fraga is funded by a PhD scholarship from the Science without Borders program - CNPq Brazil - INAGEMP/ Grant CNPq 573993/2008-4. Alex J. Diamond is funded by a BBSRC DTP PhD Scholarship. 
2
Beedie S, Peer CJ, Pisle S et al. Anti-cancer properties of a novel class of tetrafluorinated thalidomide analogs. Molecular Cancer Therapeutics In Press

D'Amato RJ, Loughnan M, Flynn E and Folkman J (1994) Thalidomide is an inhibitor of angiogenesis. Proc Natl Acad Sci U S A 91(9): 4082-4085.

DiPaolo JA, Gatzek H and Pickren J (1964) Malformations Induced in the Mouse by Thalidomide. Anat Rec 149: 149-155.

El-Aarag, BYA et al. (2014) In vitro anti-proliferative and antiangiogenic activities of thalidomide dithiocarbamate analogs. Int Immunopharmacol 21(2): 283.

Ema M, Ise R, Kato $\mathrm{H}$ et al. (2010) Fetal malformations and early embryonic gene expression response in cynomolgus monkeys maternally exposed to thalidomide. Reprod Toxicol 29(1): 49-56.

Fort DJ, Stover EL, Bantle J and Finch RA (2000) Evaluation of the developmental toxicity of thalidomide using frog embryo teratogenesis assay-xenopus (FETAX): biotransformation and detoxification. Teratog Carcinog Mutagen 20(1): 35-47.

Franks ME, Macpherson GR and Figg WD (2004) Thalidomide. Lancet 363(9423): 1802-1811.

Fratta ID, Sigg EB and Maiorana K (1965) Teratogenic Effects of Thalidomide in Rabbits, Rats, Hamsters, and Mice. Toxicol Applied Pharmacol 7: 268.

Gao X, Yourick JJ and Sprando RL (2014) Transcriptomic characterization of C57BL/6 mouse embryonic stem cell differentiation and its modulation by developmental toxicants. PLoS One 9(9):e108510.

Gao X, Sprando RL and Yourick JJ (2015) Thalidomide induced early gene expression perturbations indicative of human embryopathy in mouse embryonic stem cells. Toxicol Appl Pharmacol 287(1): 43-51.

Hansen JM and Harris C (2004) A novel hypothesis for thalidomide-induced limb teratogenesis: redox misregulation of the NF-kappaB pathway. Antioxid Redox Signal 6(1): 1 .

Higgins JJ, Pucilowska J, Lombardi RQ and Rooney JP (2004) A mutation in a novel ATP-dependent Lon protease gene in a kindred with mild mental retardation. Neurology 63(10): 1927.

Ito $\mathrm{T}$, Ando $\mathrm{H}$ and Handa $\mathrm{H}$ (2011) Teratogenic effects of thalidomide: molecular mechanisms. Cell Mol Life Sci 68(9): 1569.

Ito T, Ando H, Suzuki T et al. (2010) Identification of a primary target of thalidomide teratogenicity. Science 327(5971): 1345-1350. 
1

2

3

4

5

6

7

Knobloch J, Shaughnessy Jr, JD and Ruther U (2007) Thalidomide induces limb deformities by perturbing the Bmp/Dkk1/Wnt signaling pathway. FASEB $J$ 21(7): 1410.

Latif T, Chauhan N, Khan R, Moran A and Usmani SZ (2012) Thalidomide and its analogs in the treatment of Multiple Myeloma. Exp Hematol Oncol 1(1): 27.

Lebrin, F., Srun, S., Raymond, K et al. (2010) Thalidomide stimulates vessel maturation and reduces epistaxis in individuals with hereditary hemorrhagic telaniectasia. Nature Medicine 16: 420-428

Lee KM, Yang SJ, Kim YD et al. (2013) Disruption of the cereblon gene enhances hepatic AMPK activity and prevents high-fat diet-induced obesity and insulin resistance in mice. Diabetes 62:1855-1864.

Lenz W and Knapp K (1962) Foetal malformations due to thalidomide. German Med. Monthly 7: 253-258.

Lenz W (1988) A short history of thalidomide embryopathy. Teratology 38(3): 203215.

Lenz W, Pfeiffer RA, Kosenow K and Hayman DJ (1962) Thalidomide and Congenital Abnormalities. Lancet 279(7219): 45.

Lu J, Helsby N, Palmer BD et al. (2004) Metabolism of thalidomide in liver microsomes of mice, rabbits, and humans. J Pharmacol Exp Ther 310(2): 571.

Mahony C, Erskine L, Niven J et al. (2013) Pomalidomide is nonteratogenic in chicken and zebrafish embryos and nonneurotoxic in vitro. Proc Natl Acad Sci U S A 110(31): 12703.

Majumdar S, Lamothe B and Aggarwal BB (2002) Thalidomide suppresses NFkappa $B$ activation induced by TNF and $\mathrm{H} 2 \mathrm{O} 2$, but not that activated by ceramide, lipopolysaccharides, or phorbol ester. J Immunol 168(6): 2644-2651.

Majumder S, Rajaram M, Muley A et al. (2009) Thalidomide attenuates nitric oxidedriven angiogenesis by interacting with soluble guanylyl cyclase. Br J Pharmacol 158(7): 1720.

Marks MG, Shi J, Fry MO et al. (2002) Effects of putative hydroxylated thalidomide metabolites on blood vessel density in the chorioallantoic membrane (CAM) assay and on tumor and endothelial cell proliferation. Biol Pharm Bull 25(5): 597-604.

Matthews SJ and McCoy C (2003) Thalidomide: a review of approved and investigational uses. Clin Ther 25(2): 342-395.

McBride WG (1961) Thalidomide and Congenital Abnormalities. Lancet 278(7216): 1358. 
1 McCredie J (2009) History, heresy and radiology in scientific discovery. J Med

2 Imaging Radiat Oncol 53(5): 433-441.

3 Meganathan K, Jagtap S, Wagh V et al. (2012) Identification of thalidomide-specific

4 transcriptomics and proteomics signatures during differentiation of human embryonic

5 stem cells. PloS One 7(8): e44228.

6 Melchert M and List A (2007) The thalidomide saga. Int J Biochem Cell Biol 39(7-8):

7 1489-1499.

8 Miller MT, Stromland K, Ventura L et al. (2005) Autism associated with conditions

9 characterized by developmental errors in early embryogenesis: a mini review. Int $J$

10 Dev Neurosci 23(2-3): 201.

Moreira AL, Sampaio EP, Zmuidzinas A et al. (1993) Thalidomide exerts its inhibitory action on tumor necrosis factor alpha by enhancing mRNA degradation. $J$ Exp Med 177(6): 1675-1680.

Newman CG (1986) The thalidomide syndrome: risks of exposure and spectrum of malformations. Clinics in perinatology 13(3): 555.

Parkhie M and Webb M (1983) Embryotoxicity and teratogenicity of thalidomide in rats. Teratology $27(3): 327$.

Parman T, Wiley MJ and Wells PG (1999) Free radical-mediated oxidative DNA damage in the mechanism of thalidomide teratogenesis. Nature Medicine 5:582-585.

Rashid A, Kuppa A, Kunwar A and Panda D (2015) Thalidomide (5HPP-33) suppresses microtubule dynamics and depolymerizes the microtubule network by binding at the vinblastine binding site on tubulin. Biochemistry 54(12): 2149-2159.

Ruffing L (1977) Evalulation of thalidomide children. Birth Defects Orig Artic Ser 13(1): 287-300.

Sampaio EP, Kaplan G, Miranda A et al. (1993) The influence of thalidomide on the clinical and immunologic manifestation of erythema nodosum leprosum. $J$ Infect Dis 168(2): 408-414.

Schmidt M and Salzano FM (1980) Dissimilar effects of thalidomide in dizygotic twins. Acta Genet Med Gemellol (Roma) 29(4): 295-297.

Schuster SR, Kortuem KM, Zhu YX et al. (2014) The clinical significance of cereblon expression in multiple myeloma. Leuk Res 38(1): 23.

Sheskin J (1965) Thalidomide in the treatment of lepra reactions. Clin Pharmacol Ther 6: 303-306.

Siamwala JH, Veeriah V, Priya MK et al. (2012) Nitric oxide rescues thalidomide mediated teratogenicity. Sci Rep 2: 679. 
1

2

3

4

5

6

7

8

Smithells RW and Newman CG (1992) Recognition of thalidomide defects. J Med

Gen 29(10): 716.

Stephens TD (2009) The effect of thalidomide in chicken embryos. Birth Defects Res A Clin Mol Teratol 85(8): 725.

Stewart AK (2014) Medicine. How thalidomide works against cancer. Science 343(6168): 256.

Tamilarasan KP, Kolluru GK, Rajaram M et al. (2006) Thalidomide attenuates nitric oxide mediated angiogenesis by blocking migration of endothelial cells. BMC Cell Biol 7: 17.

Therapontos C, Erskine L, Gardner ER et al. (2009) Thalidomide induces limb defects by preventing angiogenic outgrowth during early limb formation. Proc Natl Acad Sci U S A 106(21): 85738.

Uhl K, Cox E, Rogan R et al. (2006) Thalidomide use in the US : experience with pregnancy testing in the S.T.E.P.S. programme. Drug Saf 29(4): 321-329.

Vargesson N (2003) Vascularisation of the developing chick lim bud: role of the TGFbeta signalling pathway. J. Anat 202:93-103.

Vargesson N (2009) Thalidomide-induced limb defects: resolving a 50-year-old puzzle. Bioessays 31(12): 1327-1336.

Vargesson N (2013) Thalidomide Embryopathy: An Enigmatic Challenge. ISRN Developmental Biology Article ID 241016: 18 pages. 2013. http://dx.doi.org/2010.1155/2013/241016.

Vargesson N (2015) Thalidomide-induced teratogenesis: History and mechanisms. Birth Defects Research Part C: Embryo Today: Reviews 105: 140-156

Vargesson N and Laufer E (2001) Smad7 misexpression during embryonic angiogenesis causes vascular dilation and malformations independently of vascular smooth muscle cell function. Dev Biol 240(2): 499-516.

Vianna FS, Schuler-Faccini L, Leite JC et al. (2013) Recognition of the phenotype of thalidomide embryopathy in countries endemic for leprosy: new cases and review of the main dysmorphological findings. Clin Dysmorphol 22(2): 59.

Xin W, Xiaohua N, Peilin C et al. (2008) Primary function analysis of human mental retardation related gene CRBN. Mol Biol Rep 35(2): 251. 
1 Yabu T, Tomimoto H, Taguchi $Y$ et al. (2005) Thalidomide-induced antiangiogenic

2 action is mediated by ceramide through depletion of VEGF receptors, and is

3 antagonized by sphingosine-1-phosphate. Blood 106(1): 125-134.

4

5

6

7

$8 \quad$ Further Reading

9 Magazanik M (2015) Silent Shock: The Men Behind the Thalidomide Scandal and an 10 Australian Family's Long Road to Justice. Melbourne: Text Publishing.

11

12

Stephens TD, Bunde CJJ and Fillmore BJ (2000) Mechanisms of action in thalidomide teratogenesis. Biochemical Pharmacology 59(12):1489-1499.

Vargesson N (2011) Thalidomide. Reproductive and Developmental Toxicology; Editor: R Gupta; pp395-403. Amsterdam: Academic Press Elsevier.

Zuniga A, Zeller R and Probst S (2012) The molecular basis of human congenital limb malformations. Wiley Interdisciplinary Reviews: Developmental Biology 1(6):803-822. 


\section{$1 \quad$ List of Abbreviations}

2

3 BMP - bone morphogenetic protein

$4 \quad$ cGMP - cyclic guanosine monophosphate

5 COX2 - cyclooxygenase 2

6 CUL4A - cullin 4A

7 DDB1 - DNA damage-binding protein 1

8 ENL - erythema nodosum leprosum

9 FDA - US Food and Drug Administraion

10 FGF -fibroblast growth factor

$11 \mathrm{HHT}$ - hereditary hemorrhagic telangiectasia

12 HUVEC - human umbilical vein endothelial cells

13 IL - interleukin

$14 \mathrm{MM}$ - multiple myeloma

15 NF-kB - nuclear factor - kappa beta

$16 \mathrm{NO}$ - nitric oxide

17 Roc1 - Regulator of cullin1

18 ROS - reactive oxygen species

19 SGC - soluble guanylyl cyclase

20 Shh - sonic hedgehog

21 TNFa - tumour necrosis factor alpha

22 VEGF - vascular endothelial growth factor 
Table 1: A list of common defects seen in TE, the specific defects seen in each organ, and an explanation of each. Also listed are the time points at which thalidomide is taken that can result in each defect.

\begin{tabular}{|c|c|c|c|}
\hline $\begin{array}{l}\text { Region } \\
\text { Affected }\end{array}$ & Specific Defects Seen & Additional & $\begin{array}{c}\text { Time Point of Exposure } \\
\text { (Days) }\end{array}$ \\
\hline \multirow[t]{6}{*}{ Limb } & Reduced hand/footplate & Digit effects & Thumb aplasia: $21-26$ \\
\hline & & & Triphalangeal thumb: 31-36 \\
\hline & Amelia & Complete absence of limb & Upper limb: 24-29 \\
\hline & & & Lower limb: 27-31 \\
\hline & Phocomelia & Limb long bones are & Upper limb: 24-33 \\
\hline & & shortened or absent & Lower limb: 28-33 \\
\hline \multirow[t]{5}{*}{$\begin{array}{l}\text { Limb } \\
\text { Girdles }\end{array}$} & Sharpened shoulder & $\begin{array}{l}\text { Acromioclavicular joint is more } \\
\text { prominent }\end{array}$ & \\
\hline & Hip joint / Pubic bone & Hypoplasia & Hip dislocation: $23-34$ \\
\hline & & & Femoral hip hypoplasia: 28-33 \\
\hline & & & Hip dysplasia: $20-24$ \\
\hline & & Complete absence & \\
\hline \multirow[t]{7}{*}{ Eye } & Cataracts & & $20-24$ \\
\hline & Microphthlamia & Congenital small eye & $24-30$ \\
\hline & Anophthalmos & Absence of eyeball & \\
\hline & Poor vision & & \\
\hline & Aberrant lacrimation & 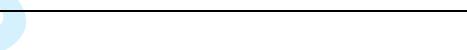 & $20-26$ \\
\hline & Colobomas & Derformity of iris and retina & $24-26$ \\
\hline & $\begin{array}{l}\text { Abnormalities in eye } \\
\text { movement }\end{array}$ & $\sqrt{2 y}$ & \\
\hline \multirow[t]{3}{*}{ Ear } & Anotia & Complete absence of outer & $20-24$ \\
\hline & & ear, results in deafness & Inner ear defects: $24-33$ \\
\hline & Microtia & Part of the outer ear remains & $24-33$ \\
\hline \multirow[t]{14}{*}{$\begin{array}{l}\text { Internal } \\
\text { organs }\end{array}$} & Heart & $\begin{array}{l}\text { Ventricular and atrial septum } \\
\text { defects }\end{array}$ & $22-31$ \\
\hline & & Pulmonary stenosis & \\
\hline & & Patent ductus arteriosus & \\
\hline & Lung & Lung malformation & $29-32$ \\
\hline & Kidney & $\begin{array}{l}\text { Horseshoe, hypoplastic, } \\
\text { rotated and ecoptic } \\
\text { malformations }\end{array}$ & Ectopic kidney: $24-29$ \\
\hline & Intestines & Duodenum & Duodenal atresia: $20-33$ \\
\hline & & & Duodenal stenosis: $27-34$ \\
\hline & & Anal atresia & Anal atresia: $27-29$ \\
\hline & & & Rectal stenosis: 35-36 \\
\hline & & Gall bladder atresia & $28-29$ \\
\hline & & Pyloric stenosis & $26-33$ \\
\hline & Urinary tract & Bladder atresia & $28-29$ \\
\hline & Genitals & $\begin{array}{l}\text { In males: absence of testes or } \\
\text { testicular abnormalities. } \\
\text { Hypospadias }\end{array}$ & Testicular agenesis: $31-33$ \\
\hline & & $\begin{array}{l}\text { In females: malformations of } \\
\text { uterus and reproductive tract }\end{array}$ & $35-39$ and $49-50$ \\
\hline \multirow{2}{*}{$\begin{array}{l}\text { Nerves and } \\
\text { CNS }\end{array}$} & Facial palsies & Ear defects are associated & Facial palsy: $20-26$ \\
\hline & & with cranial nerve palsies & Cranial palsy: 21-23 \\
\hline
\end{tabular}




\section{Figure Legends}

2

3 Figure 1: Structures of thalidomide and its analogs Thalidomide enantiomers $\mathrm{R}$

$4 \quad(+)$ and $\mathrm{S}(-)$ can interchange at physiological $\mathrm{pH}$ (asterisk indicates chiral centre).

5 Figure 2: Therapeutic mechanisms of thalidomide in adults. Illustrated are the

6 pathways through which thalidomide is thought to act in the treatment of HHT and

$7 \quad$ Multiple Myeloma. (Adapted from Stewart, 2014; Lebrin et al., 2010)

8 Figure 3: Thalidomide time-sensitive window. Chart indicates the period (days 9 and weeks post-fertilisation) in which the most common defects occur. See also 10 Table 1. (Adapted from Vargesson, 2015; Miller et al., 2005).

11 Figure 4: Thalidomide and embryonic teratogenesis. Thalidomide has been shown to induce loss of blood vessels, increased cell death and reactive oxygen species resulting in embryonic damage. Thalidomide may cause teratogenesis through interaction with targets such as Cereblon, tubulin and/or sGC, interrupting blood vessel development and resulting in localised reactive oxygen species and cell death induction. 

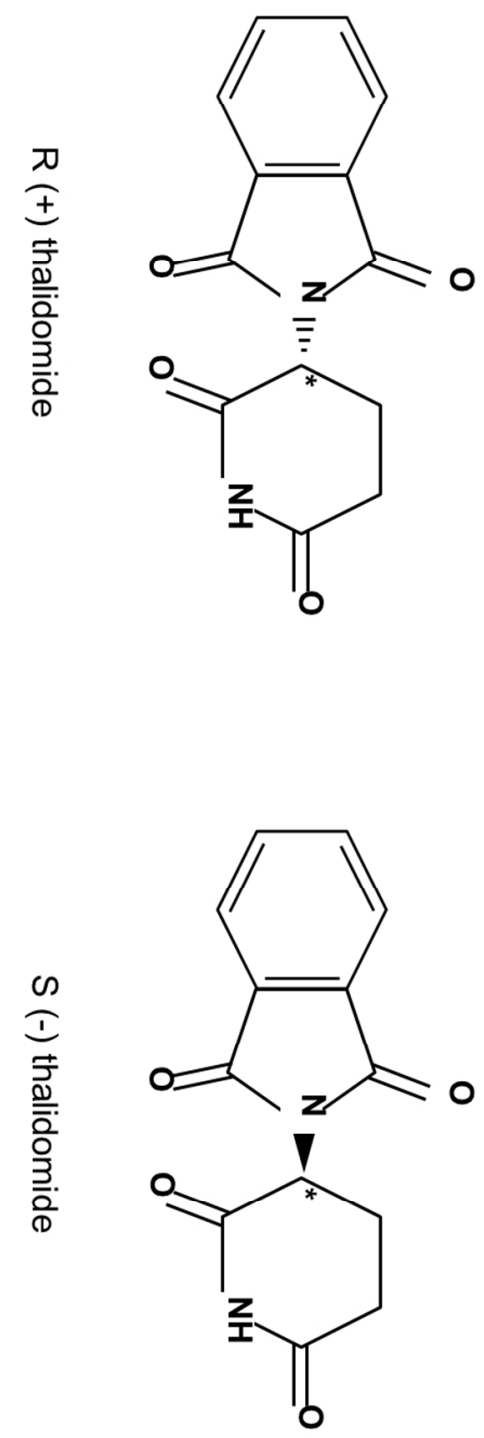

Figure 1

266×382mm (96 x 96 DPI) 


\section{Therapeutic Mechanisms of Thalidomide in the Adult}

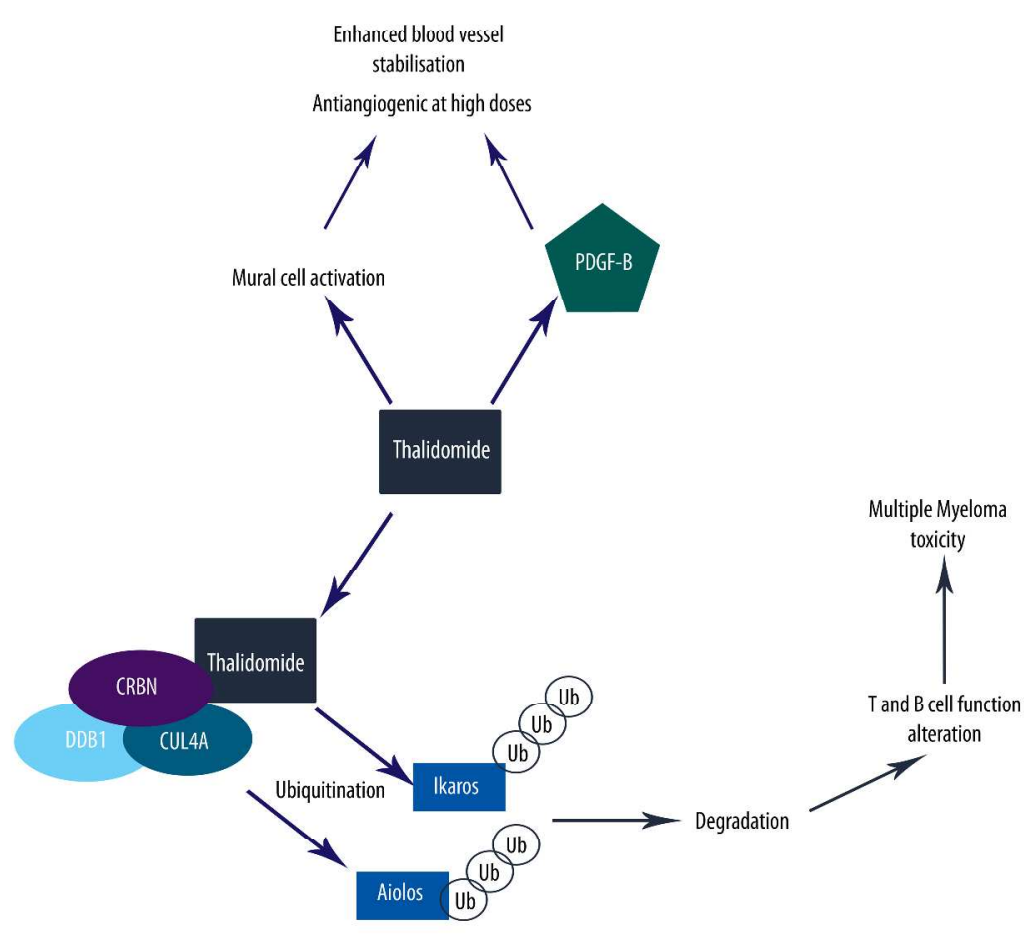

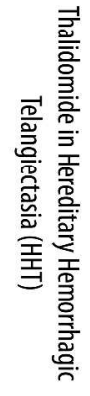

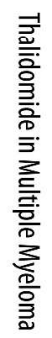

$444 \times 366 \mathrm{~mm}(300 \times 300$ DPI $)$ 


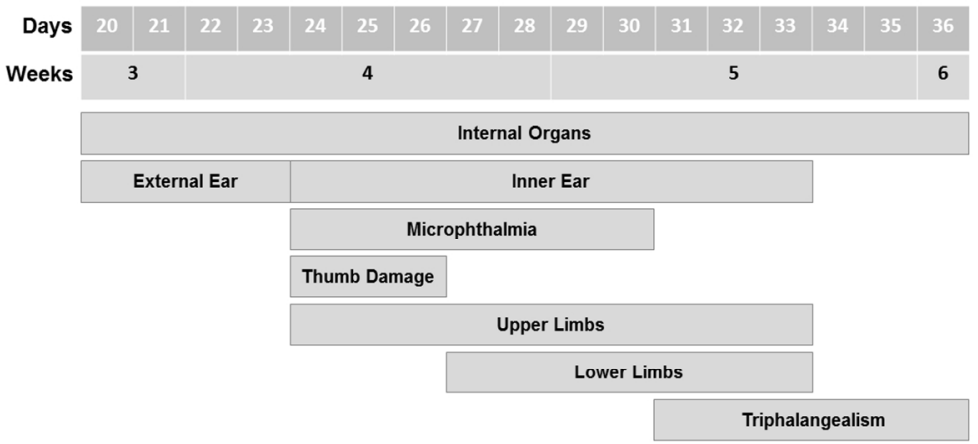

Figure 3

$382 \times 266 \mathrm{~mm}(96 \times 96 \mathrm{DPI})$ 


\section{Thalidomide and Embryonic Teratogenesis}

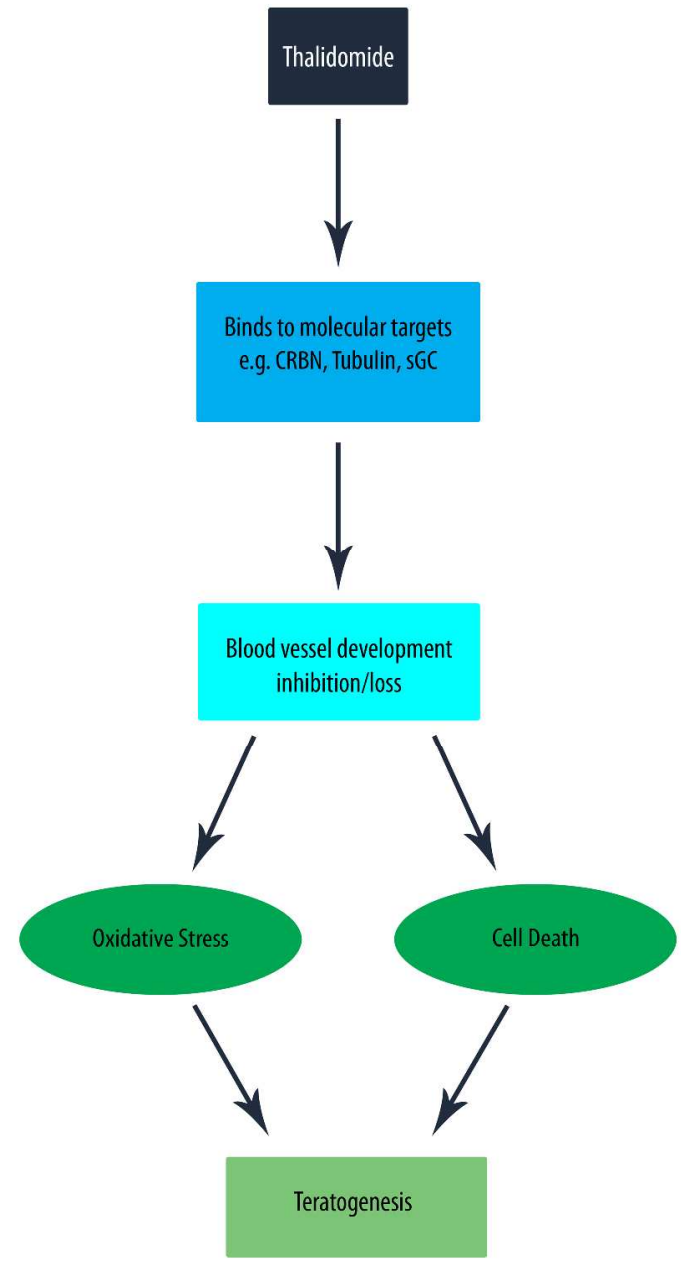

$277 \times 466 \mathrm{~mm}(300 \times 300$ DPI $)$ 